\title{
Some Characterizations of the Distribution of the Condition Number of a Complex Gaussian Matrix
}

https://doi.org/10.1515/spma-2020-0004

Received September 21, 2019; accepted November 26, 2019

Abstract: The objective of this paper is to characterize the distribution of the condition number of a complex Gaussian matrix. Several new distributional properties of the distribution of the condition number of a complex Gaussian matrix are given. Based on such distributional properties, some characterizations of the distribution are given by truncated moment, order statistics and upper record values.

Keywords: Characterization, Condition number, Gaussian matrices, Order statistics, Truncated first moment, Upper record values

MSC: 15A12, 15A52

\section{Introduction}

Gaussian matrices appear in many fields of pure and applied sciences, such as, probability, statistics, multivariate statistics, linear algebra, actuarial science, physics, wireless communications, and polarimetric synthetic aperture radar (PolSAR). Many researchers have studied the distributions of the condition numbers of Gaussian and Wishart matrices, their properties and applications, for which the interested readers are referred to Demmel (1988), Edelman (1988, 1992), Chen and Dongarra (2005), Anderson and Wells (2009), Wei et al. (2011), Zhong et al. (2011), Zhang et al. (2016), and Deng et al. (2017), among others. For some related research papers, the interested readers are also referred to Lei et al. (2014), and references therein.

Edelman (1992) studied the distribution of Demmel condition number of complex Wishart matrices. Wei et al. (2011) derived the probability density function (PDF) of Demmel condition number (DCN) for complex Wishart matrices using Mellin transformation. Shakil and Ahsanullah (2016) studied the characterizations of the distribution of the Demmel condition number of real Wishart matrices. Recently, Shakil and Ahsanullah (2017) studied the characterizations of the distribution of the Demmel condition number of complex Wishart matrices. Also, Shakil and Ahsanullah (2018) studied the characterizations of the distribution of the condition numbers of real Gaussian matrices. Shi et al. (2013) studied the exact distribution of the condition number of complex random matrices. In this paper, we study some distributional properties and characterizations of the distribution of the condition numbers of complex Gaussian matrices.

The organization of this paper is as follows. In Section 2, some preliminaries are given. In Section 3, several distributional properties of the distributions of the condition numbers of complex Gaussian matrices are presented. The characterizations of the distributions of the condition numbers of complex Gaussian matrices are provided in Section 4. In Section 5, we have computed the percentage points. In Section 6, the concluding remarks are given.

`Corresponding Author: M. Shakil: Miami Dade College, Hialeah, FL 33012, USA, E-mail: mshakil@mdc.edu M. Ahsanullah: Rider University, Lawrenceville, NJ 08648, USA, E-mail: ahsan@rider.edu 


\section{Some Preliminaries}

In this section, we will first provide some preliminaries on the notions of the condition number of a matrix which will be relevant for establishing the various results of our paper. Then, a brief discussion of complex Gaussian matrix will be presented.

\subsection{Condition Number of a Matrix}

Condition number of a matrix is one of the widely used matrix features and has significant applications in many areas of numerical analysis, linear algebra, statistics, physics and engineering. The idea of the condition number of a matrix was first introduced in 1948 by Turing (1948). Further developments continued with the contributions of Rice (1966), Skeel (1979), Geurts (1982), Demmel (1987), Edelman (1988), Trefethen and Bau (1997), Bottcher and Grudsky (1998), Trefethen and Viswanath (1998), and Higham (2002), among others. For recent work on condition numbers, see, for example, Hargreaves (2004), Xu and Zhang (2004), Acosta et al. (2006), and Ern and Guermond (2006), and references therein.

\subsubsection{Definition}

For a given non-singular matrix $A \in \Re^{n \times n}$, and a matrix norm $\|$.$\| , a non-negative quantity \kappa(A)$ given by

$$
\kappa(A)=\|A\| \cdot\left\|A^{-1}\right\|
$$

is defined as the condition number of $A$ and is denoted by Cond $(A)$, see, for example, Datta (1995), and Leon (2006), among others. Since the condition number is defined in terms of a particular matrix norm, many different matrix norms may be chosen. The most frequently used norm is the $\mathrm{p}$-norms denoted as $\|A\|_{p},(p \geq$ 1). Some commonly used p-norms are maximum column-sum norm $=\|A\|_{1}=\max _{1 \leq j \leq n} \sum_{i=1}^{m}\left|a_{i j}\right|$, where $a_{i j}$ 's denote the components of the matrix $A$, maximum row-sum norm $=\|A\|_{\infty}=\max _{1 \leq i \leq m} \sum_{j=1}^{n}\left|a_{i j}\right|$, and the Hilbert or spectral norm defined as $\|A\|_{2}=\sqrt{\text { maximumeigenvalueof } A^{T} A}$, where $A^{T}$ denotes the transpose of the matrix $A$, and if $A$ is Hermitian or real and symmetric, then $\|A\|_{2}=\rho(A)$, where $\rho(A)$ denotes the spectral radius of $A$ defined as $\rho(A)=\max _{i}\left|\lambda_{i}\right|$, where $\lambda^{\prime} s$ are the eigenvalues of $A$. The condition number corresponding to the p-norm will be denoted $\kappa_{p}(A)$. For a singular square matrix $\mathrm{A}^{n \times n}$, that is, if $\mathrm{A}$ is not invertible, the condition number of $\mathrm{A}$ is infinite, that is, $\kappa(A)=+\infty$.

\subsubsection{Condition Number Based on Spectral Norm}

Let $\|$.$\| denote the spectral norm. Let A$ be a nonsingular $n \times n$ matrix. Let $\sigma_{1}, \sigma_{2}, \ldots, \sigma_{n}$ denote the $n$ singular values of $A$.

Definition 1: Let $\lambda_{1}, \lambda_{2}, \ldots, \lambda_{n}$ denote the corresponding $n$ eigenvalues of $A$. Then the condition number $\kappa_{2}(A)$ of $A$ is defined by

$$
\kappa_{2}(A)=\|A\|_{2} \cdot\left\|A^{-1}\right\|_{2}=\frac{\max _{1 \leq i \leq n}\left|\sigma_{i}\right|}{\min _{1 \leq i \leq n}\left|\sigma_{i}\right|},
$$

where $\max _{1 \leq i \leq n}\left|\sigma_{i}\right|$ and $\min _{1 \leq i \leq n}\left|\sigma_{i}\right|$ denote the largest and smallest singular values of $A$ respectively.

Definition 2. Let . denote the spectral norm. Let $A$ be a nonsingular $n \times n$ matrix. If $A$ is Hermitian or real and symmetric, then the condition number $\kappa_{2}(A)$ of $A$ is defined by

$$
\kappa_{2}(A)=\|A\|_{2} \cdot\left\|A^{-1}\right\|_{2}=\frac{\max _{1 \leq i \leq n}\left|\lambda_{i}\right|}{\min _{1 \leq i \leq n}\left|\lambda_{i}\right|},
$$


where $\max _{1 \leq i \leq n}\left|\lambda_{i}\right|$ and $\min _{1 \leq i \leq n}\left|\lambda_{i}\right|$ are, respectively, the largest and smallest eigenvalues in modulus of $A$.

Note that if the columns of $A$ are orthogonal, the condition number, $\kappa_{2}(A)$, of $A$ attains its minimum bound, that is, $\kappa_{2}(A) \geq 1$, (see, for example, Golub and Van Loan (1996), among others). If $\kappa_{2}(A)$ is large, then $A$ is said to be an ill-conditioned matrix. If $\kappa_{2}(A)$ is near unity, then $A$ is said to be a well-conditioned matrix. Matrices with small condition numbers are said to be well-conditioned.

\subsection{Complex Gaussian Matrices}

A matrix is called a random matrix if the entries of the matrix are random numbers from a specified distribution. If the distribution is Gaussian, then we call it a Gaussian random matrix. Consider an $m \times n$ rectangular random matrix $X$ all of whose elements are independent standard normal random variables. We call such a random matrix (or its distribution) as a Gaussian matrix, and denote it by $G(m, n)$.

A complex standard normally distributed random variable can be defined as $u+v i, i=\sqrt{-1}$, where $u$ and $v$ are independent and identically distributed (i.i.d.) standard normal random variables. If all the components of a random matrix are independent complex standard normal random variables, we call it a complex Gaussian matrix; see, for example, Haagerup, and Thorbjørnsen (2003). Let $\tilde{X}$ be such a complex Gaussian matrix of the form $X_{1}+i X_{2}$, where $X_{1}$ and $X_{2}$ are independent and have the distribution $G(m, n)$. Let $\tilde{G}(m, n)$ denote the distribution of $\tilde{X}$. Let $\tilde{X}^{T}$ denote the transpose of the $n \times n$ random matrix $\tilde{X}$. Then, the $n \times n$ random matrix $W_{n \times n}=\tilde{X} \tilde{X}^{T}$ is said to be a Wishart matrix or to have the Wishart distribution with parameters $(n, n)$. That is, $W_{n \times n}\left(=\tilde{X} \tilde{X}^{T}\right) \sim W(n, n)$.

Further, let $\lambda_{\max }=\lambda_{1}>\lambda_{2} \cdots>\lambda_{n}=\lambda_{\min }>0$ and $\sigma_{1}>\sigma_{2} \cdots>\sigma_{n}>0$ denote the distinct eigenvalues of the matrix $W_{n \times n}=\tilde{X} \tilde{X}^{T}$ and singular values of $\tilde{X}$, respectively. Note that the squares of the singular values of $\tilde{X}$ are the eigenvalues of the Wishart matrix $W_{n \times n}=\tilde{X} \tilde{X}^{T}$. Then, the random quantity $\kappa_{2}(\tilde{X})=\sqrt{\frac{\lambda_{1}}{\lambda_{n}}}=\sqrt{\frac{\lambda_{\max }}{\lambda_{\min }}}=\frac{\sigma_{1}}{\sigma_{n}}$, the square root of the ratio of largest to smallest eigenvalues of the Wishart matrix $W_{n \times n}=\tilde{X} \tilde{X}^{T}$, is defined as the 2-norm (or the standard) condition number of $\tilde{X}$, see, for example, Edelman (1988).

\section{Distributional Properties of the Condition Numbers of Complex Gaussian Matrices}

In this section, we discuss the properties of the distribution of the condition numbers of complex Gaussian matrices. For the sake of simplicity and without loss of generality, we shall consider the explicit expressions of the probability density function (pdf) of the exact distribution of the distribution of the condition number, $\tilde{\kappa}$, of a complex Gaussian matrix, $\tilde{X}$, having the distribution $\tilde{G}(2, n)$.

\subsection{Probability Density Function}

Edelman (1988) introduced a distribution for a continuous random variable $X$ to study the distribution $\tilde{G}(2, n)$ of the condition number, $\tilde{\kappa}$, of a complex Gaussian matrix, $\tilde{X}$, with the probability density function (pdf), $f(x)$, given by

$$
f(x)=\frac{2 \Gamma(2 n)}{\Gamma(n) \Gamma(n-1)} \frac{x^{2 n-3}\left(x^{2}-1\right)^{2}}{\left(x^{2}+1\right)^{2 n}}, x>1, n \geq 2 .
$$


By using the substitution $t^{2}+1=\frac{1}{u}$, the corresponding cumulative distribution function (cdf) is easily derived as follows:

$$
\begin{aligned}
F(x) & =\int_{1}^{x} \frac{2 \Gamma(2 n)}{\Gamma(n) \Gamma(n-1)} \frac{t^{2 n-3}\left(t^{2}-1\right)^{2}}{\left(t^{2}+1\right)^{2 n}} d t \\
& =1-\frac{\Gamma(2 n)}{\Gamma(n) \Gamma(n-1)}\left[B_{1 /\left(1+x^{2}\right)}(n-1, n-1)-4 B_{1 /\left(1+x^{2}\right)}(n, n)\right],
\end{aligned}
$$

where $B_{x}(a, b)=\int_{0}^{x} u^{a-1}(1-u)^{b-1} d u$ denotes the incomplete beta function, see, for example, Abramowitz and Stegun (1970), and Gradshteyn and Ryzhik (1990). For some selected values of the parameter, $n$, the graphs of $f(x)$ and $F(x)$ are given in Figures 1 and 2 respectively. The effects of the parameters are obvious from these figures, that is, the distribution appears to be unimodal and right skewed.

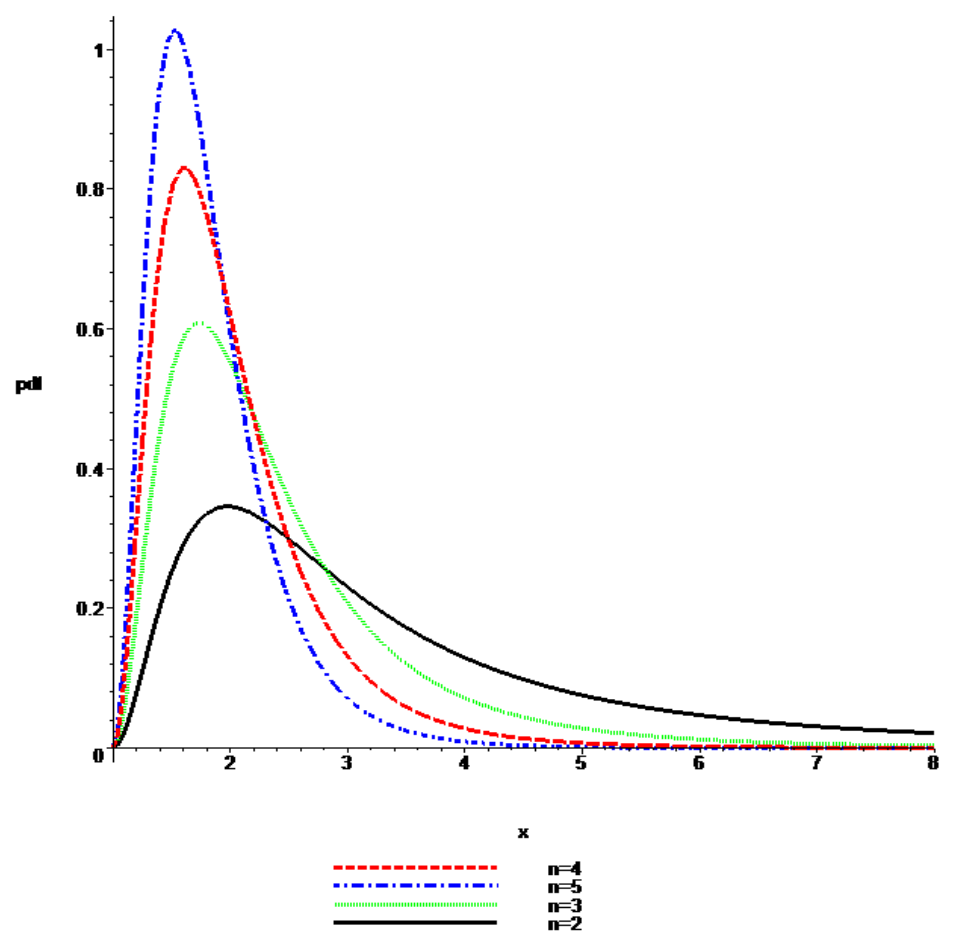

Figure 1: Plots of the pdf (3.3) for $n=2,3,4,5$

\subsection{Survival and Hazard Functions}

The survival and hazard functions are respectively given by

$$
S(x)=1-F_{X}(x)=\frac{\Gamma(2 n)}{\Gamma(n) \Gamma(n-1)}\left[B_{1 /\left(1+x^{2}\right)}(n-1, n-1)-4 B_{1 /\left(1+x^{2}\right)}(n, n)\right],
$$

and

$$
h(x)=\frac{f_{X}(x)}{1-F_{X}(x)}=\frac{2 x^{2 n-3}\left(x^{2}-1\right)^{2}}{\left[B_{1 /\left(1+x^{2}\right)}(n-1, n-1)-4 B_{1 /\left(1+x^{2}\right)}(n, n)\right]\left(x^{2}+1\right)^{2 n}}
$$

where $x>1, n \geq 2$. The possible shapes of $h(x)$ are provided for some selected values of the parameter, $n$, in the Figure 3. The effects of the parameters can easily be seen from these graphs. The upside down bathtub shape behaviors of $h(x)$ are evident from the figure. 


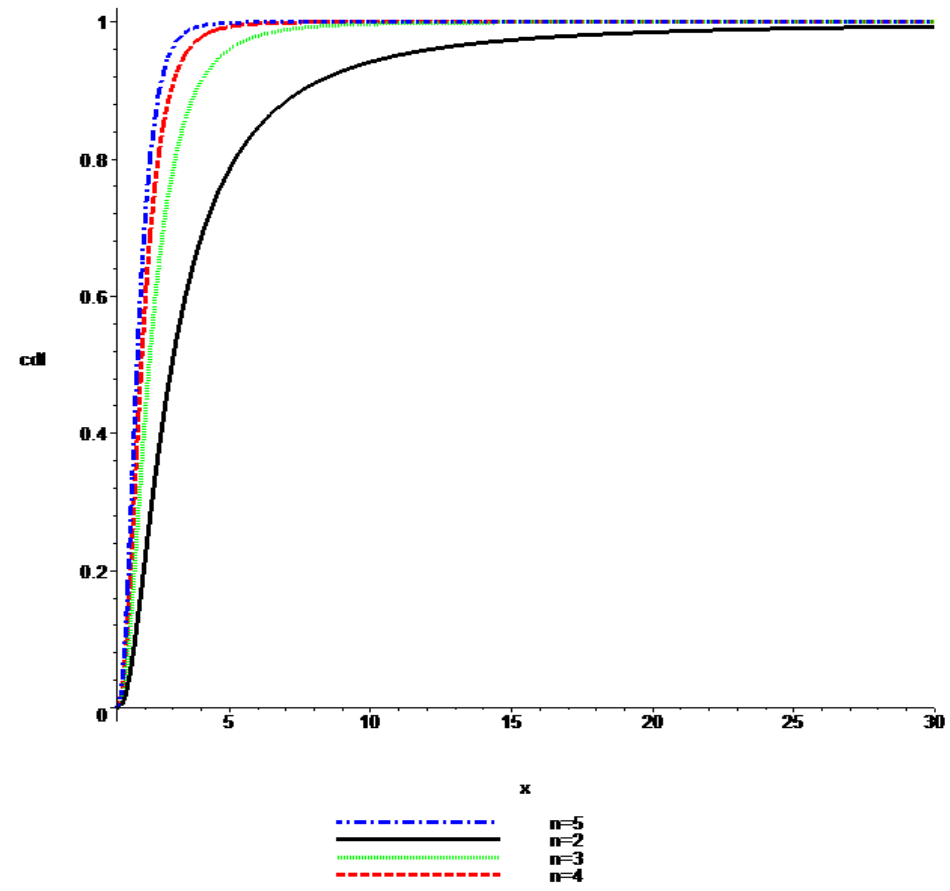

Figure 2: Plots of the $\operatorname{cdf}(3.2)$ for $n=2,3,4,5$

\subsection{Moment}

The $j$ th moment, $E\left(X^{j}\right)$, where $j>0$ is an integer is given by

$$
E\left(X^{j}\right)=\int_{1}^{\infty} x^{j} f(x) d x=\int_{1}^{\infty} \frac{2 \Gamma(2 n)}{\Gamma(n) \Gamma(n-1)} \frac{x^{2 n+j-3}\left(x^{2}-1\right)^{2}}{\left(x^{2}+1\right)^{2 n}} d x,
$$

from which on substituting $x^{2}+1=\frac{1}{u}$, we obtain

$$
\begin{aligned}
E\left(X^{j}\right) & =\int_{0}^{1 / 2} \frac{\Gamma(2 n)}{\Gamma(n) \Gamma(n-1)} u^{2 n}\left(\frac{1-u}{u}\right)^{n-2+\frac{j}{2}}\left(\frac{1-2 u}{u}\right)^{2} \frac{1}{u^{2}} d u \\
& =\frac{\Gamma(2 n)}{\Gamma(n) \Gamma(n-1)} \int_{0}^{1 / 2} u^{n-2-\frac{j}{2}}(1-u)^{n-2+\frac{j}{2}}[1-4 u(1-u)] d u \\
& =\frac{\Gamma(2 n)}{\Gamma(n) \Gamma(n-1)}\left[B_{1 / 2}\left(n-\frac{j}{2}-1, n+\frac{j}{2}-1\right)-4 B_{1 / 2}\left(n-\frac{j}{2}, n+\frac{j}{2}\right)\right],
\end{aligned}
$$

since $B_{x}(a, b)=\int_{0}^{x} u^{a-1}(1-u)^{b-1} d u$. When $j=1$ in Eq. (3.5), the first moment is given by

$$
E(X)=\frac{\Gamma(2 n)}{\Gamma(n) \Gamma(n-1)}\left[B_{1 / 2}\left(n-\frac{3}{2}, n-\frac{1}{2}\right)-4 B_{1 / 2}\left(n-\frac{1}{2}, n+\frac{1}{2}\right)\right],
$$

For some values of the parameter, $n \geq 2$, the first moment, $E(X)$, is tabulated from Eq. (3.6), in Table 1.

\subsection{Entropy}

The entropy measure of a random variable $X$ is a measure of variation of uncertainty and has been used in many fields such as physics, engineering and economics, among others. According to Shannon (1948), the 


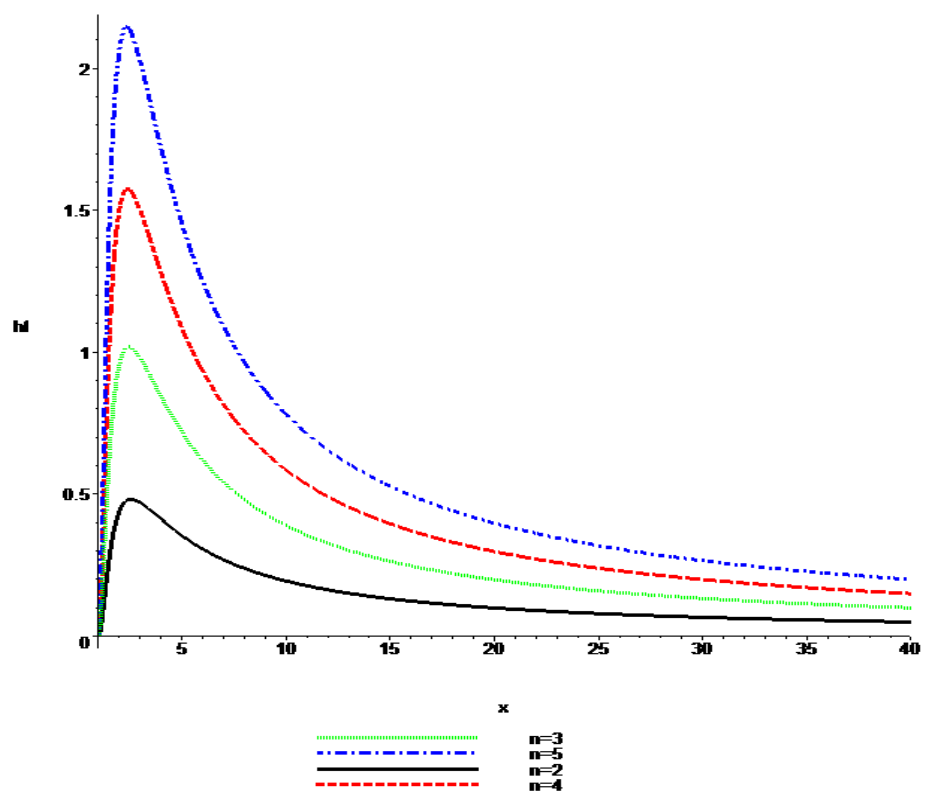

Figure 3: Plots of the hazard rate function, $h(x)$, for $n=2,3,4,5$

Table 1: Table for the first moment, $E(X)$

\begin{tabular}{cc}
\hline$n$ & $E(X)$ \\
\hline 2 & 4.3562 \\
3 & 2.4726 \\
4 & 2.0385 \\
5 & 1.8330 \\
6 & 1.7096 \\
7 & 1.6258 \\
8 & 1.5646 \\
9 & 1.5174 \\
10 & 1.4798 \\
\hline
\end{tabular}

entropy measure of a continuous real random variable $X$ is defined as

$$
H_{X}\left[f_{X}(X)\right]=E\left[-\ln \left(f_{X}(X)\right]=-\int_{-\infty}^{\infty} f_{X}(x) \ln \left[f_{X}(x)\right] d x .\right.
$$

Thus, the entropy measure of the random variable $X$ having the pdf $f(x)$ as in Eq. (3.1) is given by

$$
\begin{aligned}
& H_{X}\left[f_{X}(X)\right] \\
& =-\int_{1}^{\infty} \frac{2 \Gamma(2 n)}{\Gamma(n) \Gamma(n-1)} \frac{x^{2 n-3}\left(x^{2}-1\right)^{2}}{\left(x^{2}+1\right)^{2 n}} \ln \left[\frac{2 \Gamma(2 n)}{\Gamma(n) \Gamma(n-1)} \frac{x^{2 n-3}\left(x^{2}-1\right)^{2}}{\left(x^{2}+1\right)^{2 n}}\right] d x \\
& =2 n E\left(\ln \left(x^{2}+1\right)\right)-2 E\left(\ln \left(x^{2}-1\right)\right)-(2 n-3) E(\ln (x))-\ln \left(\frac{2 \Gamma(2 n)}{\Gamma(n) \Gamma(n-1)}\right),
\end{aligned}
$$

where $n \geq 2$, and $x>1$. It is obvious from the above that we cannot obtain a closed form expression for the Shannon entropy. For some values of the parameter $n \geq 2$, using the Maple software, the Shannon entropy is tabulated in Table 2, and the corresponding graph is sketched in Figure 4 below. From Table 2 and Figure 4, it is observed that the Shannon entropy is a monotonic decreasing and concave up function of $n$. 
Table 2: Table for the Shannon entropy

\begin{tabular}{cc}
\hline$n$ & $H_{X}\left[f_{X}(X)\right]=E\left[-\ln \left(f_{X}(X)\right]\right.$ \\
\hline 2 & 2.0416 \\
3 & 1.2307 \\
4 & 0.8380 \\
5 & 0.5853 \\
6 & 0.4012 \\
7 & 0.2574 \\
8 & 0.1399 \\
9 & 0.0411 \\
10 & -0.0442 \\
\hline
\end{tabular}

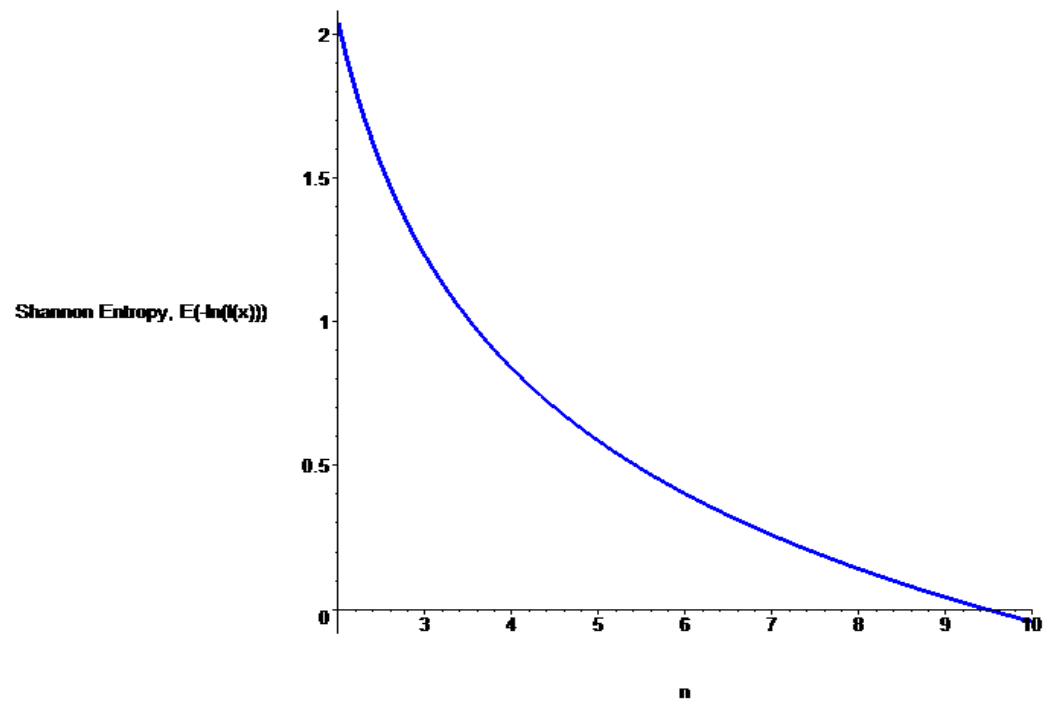

Figure 4: Plot of the Shannon entropy, $H_{X}\left[f_{X}(X)\right]=E\left[-\ln \left(f_{X}(X)\right]\right.$, for $n \geq 2$

\section{Characterization Results}

In this section, we give our proposed characterizations of the distribution of condition number of a complex Gaussian matrix by truncated moment, order statistics and upper record values.

Many authors and researchers have studied the characterizations of probability distributions, among them Galambos and Kotz (1978), Kotz and Shanbhag (1980), and Nagaraja (2006), are notable. Since the truncated distributions arise in practical statistics where the ability of record observations is limited to a given threshold or within a specified range, there has been a great interest, in recent years, in the characterizations of probability distributions by truncated moments, that is, by considering a product of reverse hazard rate and another function of the truncated point, see, for example, Ahsanullah et al. $(2015,2016)$, and Ahsanullah and Shakil (2018).

Recently, many researchers have also used the idea of truncated moment method in the studies of the characterizations of the distributions of the condition numbers (DCN) of random matrices, see, for example, Shakil and Ahsanullah (2016, 2017, 2018). It appears from the literature that no attention has been paid to the characterizations of the distribution of the condition number of a complex Gaussian matrix. Therefore, motivated by the importance of the characterizations of probability distributions in practical problems, in this section we provide some new characterization results of the distribution of the distribution of the condition 
number, $\tilde{\kappa}$, of a complex Gaussian matrix, $\tilde{X}$, having the distribution $\tilde{G}(2, n)$, with pdf (3.1) and cdf (3.2), by truncated moment, order statistics and upper record values.

For this, we will need the following assumption and lemmas. We assume that $\frac{d f(x)}{d x}$ exits for all $x$, where $1<x<\infty$. We also assume that $E(X)$ exists, which is true in our case, since the pdf (3.1) has finite $E(X)$ and, therefore, can easily be characterized using conditional expectation of the first moment.

Assumption 4.1. Suppose the random variable $X$ is absolutely continuous with the cumulative distribution function $F(x)$ and the probability density function $f(x)$. We assume that $\gamma=\inf \{x \mid F(x)>0\}$, and $\delta=\sup \{x \mid F(x)<1\}, f(x)$ is a differentiable function of $x$ in the interval $(\gamma, \delta)$, and $E(X)$ exists.

Lemma 4.1. Under the Assumption 4.1, if $E(X \mid X \leq x)=g(x) \tau(x)$, where $\tau(x)=\frac{f(x)}{F(x)}$ and $g(x)$ is a continuous differentiable function of $x$ with the condition that $\int_{\gamma}^{x} \frac{u-g^{\prime}(u)}{g(u)} d u$ is finite for $x>\gamma$, then $f(x)=c e^{\int \frac{x-g /(x)}{g(x)} d x}$, where $c$ is a constant determined by the condition $\int_{\gamma}^{\delta} f(x) d x=1$.

Proof. For proof, see Shakil and Ahsanullah (2018).

Lemma 4.2. Under the Assumption 4.1, if $E(X \mid X \geq x)=h(x) r(x)$, where $r(x)=\frac{f(x)}{1-F(x)}$ and $h(x)$ is a continuous differentiable function of $x$ with the condition that $\int_{x}^{\delta} \frac{u+h^{\prime}(u)}{h(u)} d u$ is finite for $x<\delta$, then $f(x)=c e^{\int-\left(\frac{h(x)+h /(x)}{h(x)}\right) d x}$, where $c$ is a constant determined by the condition $\int_{\gamma}^{\delta} f(x) d x=1$.

Proof. For proof, see Shakil and Ahsanullah (2018).

\subsection{Characterization by Truncated Moment}

Theorem 4.1. Suppose the random variable $X$ is absolutely continuous with the cumulative distribution function $F(x)$ and the probability density function $f(x)$. We assume that $1=\inf \{x \mid F(x)>0\}, \infty=\sup \{x \mid F(x)<1\}$, and $E(X)$ is finite for all $x$, where $1<x<\infty$. Then, $E(X \mid X \leq x)=g(x) \frac{f(x)}{F(x)}$, where

$$
\begin{aligned}
g(x) & =\frac{\left(x^{2}+1\right)^{2 n} P(x)}{2 x^{2 n-3}\left(x^{2}-1\right)^{2}}, \text { and } \\
P(x) & =\left\{\left[B_{1 / 2}\left(n-\frac{3}{2}, n+\frac{1}{2}\right)-4 B_{1 / 2}\left(n-\frac{1}{2}, n+\frac{3}{2}\right)\right] .\right. \\
& \left.-\left[B_{1 /\left(1+x^{2}\right)}\left(n-\frac{3}{2}, n+\frac{1}{2}\right)-4 B_{1 /\left(1+x^{2}\right)}\left(n-\frac{1}{2}, n+\frac{3}{2}\right)\right]\right\},
\end{aligned}
$$

if and only if

$$
f(x)=2 \frac{\Gamma(2 n)}{\Gamma(n) \Gamma(n-1)} \frac{x^{2 n-3}\left(x^{2}-1\right)^{2}}{\left(x^{2}+1\right)^{2 n}}, x>1, n \geq 2 .
$$

Proof. Necessary Part: Consider the pdf (3.1), that is,

$$
f(x)=2 \frac{\Gamma(2 n)}{\Gamma(n) \Gamma(n-1)} \frac{x^{2 n-3}\left(x^{2}-1\right)^{2}}{\left(x^{2}+1\right)^{2 n}}, x>1, n \geq 2 .
$$

Then, since $E(X \mid X \leq x)=.g(x) \tau(x)$, where $\tau(x)=\frac{f(x)}{F(x)}$, and also $E(X \mid X \leq x)=.\frac{\int_{1}^{x} u f(u) d u}{F(x)}$, we have

$$
f(x) g(x)=\int_{1}^{x} t f(t) d t=\int_{1}^{x} 2 \frac{\Gamma(2 n)}{\Gamma(n) \Gamma(n-1)} \frac{t^{2 n-2}\left(t^{2}-1\right)^{2}}{\left(t^{2}+1\right)^{2 n}} d t .
$$

Let $t^{2}+1=\frac{1}{u}$ in Eq. (4.1). Then, proceeding in the same manner as in Eq. (3.5) of sub-section 3.3, after simplification, we have

$$
\begin{aligned}
f(x) g(x) & =\frac{\Gamma(2 n)}{\Gamma(n) \Gamma(n-1)}\left\{\left[B_{1 / 2}\left(n-\frac{3}{2}, n-\frac{1}{2}\right)-4 B_{1 / 2}\left(n-\frac{1}{2}, n+\frac{1}{2}\right)\right]\right. \\
& \left.-\left[B_{1 /\left(1+x^{2}\right)}\left(n-\frac{3}{2}, n-\frac{1}{2}\right)-4 B_{1 /\left(1+x^{2}\right)}\left(n-\frac{1}{2}, n+\frac{1}{2}\right)\right]\right\}, \\
& =\frac{\Gamma(2 n)}{\Gamma(n) \Gamma(n-1)} P(x),
\end{aligned}
$$


where

$$
\begin{aligned}
P(x) & =\left\{\left[B_{1 / 2}\left(n-\frac{3}{2}, n-\frac{1}{2}\right)-4 B_{1 / 2}\left(n-\frac{1}{2}, n+\frac{1}{2}\right)\right]\right. \\
& \left.-\left[B_{1 /\left(1+x^{2}\right)}\left(n-\frac{3}{2}, n-\frac{1}{2}\right)-4 B_{1 /\left(1+x^{2}\right)}\left(n-\frac{1}{2}, n+\frac{1}{2}\right)\right]\right\} .
\end{aligned}
$$

Thus, we have

$$
g(x)=\frac{\left(x^{2}+1\right)^{2 n} P(x)}{2 x^{2 n-3}\left(x^{2}-1\right)^{2}} .
$$

This completes the proof of the necessary part.

Sufficient Part: Conversely, suppose that

$$
g(x)=\frac{P(x)\left(x^{2}+1\right)^{2 n}}{2 x^{2 n-3}\left(x^{2}-1\right)^{2}},
$$

where $P(x)$ is given by Eq. (4.3). Then, differentiating both sides of the above equation with respect to $x$, and noting that $\frac{d\left(B_{x}(a, b)\right)}{d x}=(1-u)^{b-1} u^{a-1}$, we obtain

$$
\begin{aligned}
g^{\prime}(x) & =x-\frac{P(x)\left(x^{2}+1\right)^{2 n}}{2 x^{2 n-3}\left(x^{2}-1\right)^{2}}\left(\frac{2 n-3}{x}+\frac{4 x}{x^{2}-1}-\frac{4 n x}{x^{2}+1}\right) \\
& =x-g(x)\left(\frac{2 n-3}{x}+\frac{4 x}{x^{2}-1}-\frac{4 n x}{x^{2}+1}\right),
\end{aligned}
$$

from which, we get

$$
\frac{x-g^{\prime}(x)}{g(x)}=\left(\frac{2 n-3}{x}+\frac{4 x}{x^{2}-1}-\frac{4 n x}{x^{2}+1}\right) .
$$

Since, by Lemma 4.1, we have $\frac{x-g^{\prime}(x)}{g(x)}=\frac{f^{\prime}(x)}{f(x)}$, therefore, we have

$$
\frac{f^{\prime}(x)}{f(x)}=\left(\frac{2 n-3}{x}+\frac{4 x}{x^{2}-1}-\frac{4 n x}{x^{2}+1}\right) .
$$

On integrating both sides of the above equation with respect to $x$, we obtain

$$
\ln f(x)=\ln (c)+(2 n-3) \ln (x)+2 \ln \left(x^{2}-1\right)-2 n \ln \left(x^{2}+1\right),
$$

or,

$$
f(x)=\frac{c x^{2 n-3}\left(x^{2}-1\right)^{2}}{\left(x^{2}+1\right)^{2 n}},
$$

where $c$ is a constant to be determined. Now, integrating the Eq. (4.4) with respect to $x$ from 1 to $\infty$, using the boundary condition $\int_{1}^{\infty} f(x) d x=1$, we obtain $c=\frac{2 \Gamma(2 n)}{\Gamma(n) \Gamma(n-1)}$. Then, we have

$$
f(x)=\frac{2 \Gamma(2 n)}{\Gamma(n) \Gamma(n-1)} \frac{x^{2 n-3}\left(x^{2}-1\right)^{2}}{\left(x^{2}+1\right)^{2 n}}, x>1, n \geq 2 .
$$

This completes the proof of Theorem 4.1.

Theorem 4.2. Suppose the random variable $X$ is absolutely continuous with the cumulative distribution function $F(x)$ and the probability density function $f(x)$. We assume that $1=\inf \{x \mid F(x)>0\}, \infty=\sup \{x \mid F(x)<1\}$, and $E(X)$ is finite for all $x$, where $1<x<\infty$. Then, $E(X \mid X \geq x)=h(x) r(x)$, where $r(x)=\frac{f(x)}{1-F(x)}$ and

$$
h(x)=\frac{\Gamma(n) \Gamma(n-1)\left(x^{2}+1\right)^{2 n}\left[E(x)-\frac{\Gamma(2 n)}{\Gamma(n) \Gamma(n-1)} P(x)\right]}{2 \Gamma(2 n) x^{2 n-3}\left(x^{2}-1\right)},
$$

if and only if

$$
f(x)=2 \frac{\Gamma(2 n)}{\Gamma(n) \Gamma(n-1)} \frac{x^{2 n-3}\left(x^{2}-1\right)^{2}}{\left(x^{2}+1\right)^{2 n}}, x>1, n \geq 2,
$$

where $E(x)$ denotes the first moment given by Eq. (3.6), and $P(x)$ is given by Eq. (4.3). 
Proof. Necessary Part: Consider the pdf (3.1), that is,

$$
f(x)=2 \frac{\Gamma(2 n)}{\Gamma(n) \Gamma(n-1)} \frac{x^{2 n-3}\left(x^{2}-1\right)^{2}}{\left(x^{2}+1\right)^{2 n}}, x>1, n \geq 2 .
$$

Then, since $E(X \mid X \geq x)=h(x) r(x)$, where $r(x)=\frac{f(x)}{1-F(x)}$, and also since $E(X \mid X \geq x)=\frac{\int_{x}^{\infty} u f(u) d u}{f(x)}$, we have

$$
f(x) h(x)=\int_{x}^{\infty} u f(u) d u=E(x)-\int_{1}^{x} u f(u) d u=E(x)-\frac{\Gamma(2 n)}{\Gamma(n) \Gamma(n-1)} P(x),
$$

where $E(x)$ denotes the first moment given by Eq. (3.6), and $P(x)$ is given by Eq. (4.3). It follows from (4.5) that

$$
h(x)=\frac{\Gamma(n) \Gamma(n-1)\left(x^{2}+1\right)^{2 n}\left[E(x)-\frac{\Gamma(2 n)}{\Gamma(n) \Gamma(n-1)} P(x)\right]}{2 \Gamma(2 n) x^{2 n-3}\left(x^{2}-1\right)} .
$$

This completes the proof of the necessary part.

Sufficient Part: Conversely, suppose that

$$
h(x)=\frac{\Gamma(n) \Gamma(n-1)\left(x^{2}+1\right)^{2 n}\left[E(x)-\frac{\Gamma(2 n)}{\Gamma(n) \Gamma(n-1)} P(x)\right]}{2 \Gamma(2 n) x^{2 n-3}\left(x^{2}-1\right)} .
$$

Then, differentiating both sides of the above equation with respect to $x$, and simplifying, we easily obtain

$$
h^{\prime}(x)=-x-h(x)\left(\frac{2 n-3}{x}+\frac{4 x}{x^{2}-1}-\frac{4 n x}{x^{2}+1}\right),
$$

or,

$$
\frac{x+h^{\prime}(x)}{h(x)}=-\left(\frac{2 n-3}{x}+\frac{4 x}{x^{2}-1}-\frac{4 n x}{x^{2}+1}\right) .
$$

Since, by Lemma 4.2, we have

$$
\frac{x+h^{\prime}(x)}{h(x)}=-\frac{f^{\prime}(x)}{f(x)}
$$

therefore (4.7) reduces to

$$
\frac{f^{\prime}(x)}{f(x)}=\left(\frac{2 n-3}{x}+\frac{4 x}{x^{2}-1}-\frac{4 n x}{x^{2}+1}\right) .
$$

On integrating both sides of the above Eq. (4.8) with respect to $x$, we obtain

$$
\ln f(x)=\ln (c)+(2 n-3) \ln (x)+2 \ln \left(x^{2}-1\right)-2 n \ln \left(x^{2}+1\right),
$$

or,

$$
f(x)=\frac{c x^{2 n-3}\left(x^{2}-1\right)^{2}}{\left(x^{2}+1\right)^{2 n}},
$$

where $c$ is a constant to be determined. Now, integrating the Eq. (4.9) with respect to $x$ from 1 to $\infty$, and using the boundary condition $\int_{1}^{\infty} f(x) d x=1$, we obtain $c=\frac{2 \Gamma(2 n)}{\Gamma(n) \Gamma(n-1)}$. Then, we have

$$
f(x)=\frac{2 \Gamma(2 n)}{\Gamma(n) \Gamma(n-1)} \frac{x^{2 n-3}\left(x^{2}-1\right)^{2}}{\left(x^{2}+1\right)^{2 n}}, x>1, n \geq 2 .
$$

This completes the proof of Theorem 4.2. 


\subsection{Characterizations by Order Statistics}

If $X_{1}, X_{2}, \ldots, X_{n}$ be the $n$ independent copies of the random variable $X$ with absolutely continuous distribution function $F(x)$ and pdf $f(x)$, and if $X_{1, n} \leq X_{2, n} \leq \ldots \leq X_{n, n}$ be the corresponding order statistics, then it is known from Ahsanullah, et al. (2013), chapter 5, or Arnold, et al. (2005), chapter 2, that $X_{j, n} \mid X_{k, n}=x$, for $1 \leq k<j \leq n$, is distributed as the $(j-k)$ th order statistics from $(n-k)$ independent observations from the random variable $V$ having the $\operatorname{pdf} f_{V}(v \mid x)$ where $f_{V}(v \mid x)=\frac{f(v)}{1-F(x)}, 0 \leq v<x$, and $X_{i, n} \mid X_{k, n}=x, 1 \leq i<k \leq n$, is distributed as $i$ th order statistics from $k$ independent observations from the random variable $W$ having the $\operatorname{pdf} f_{W}(w \mid x)$ where

$$
f_{W}(w \mid x)=\frac{f(w)}{F(x)}, w<x . \text { Let } S_{k-1}=\frac{1}{k-1}\left(X_{1, n}+X_{2, n}+\ldots+X_{k-1, n}\right),
$$

and

$$
T_{k, n}=\frac{1}{n-k}\left(X_{k+1, n}+X_{k+2, n}+\ldots+X_{n . n}\right) .
$$

Theorem 4.3: Suppose the random variable $X$ satisfies the Assumption 4.1 with $\gamma=1$ and $\delta=\infty$, then $E\left(S_{k-1} \mid X_{k, n}=x\right)=g(x) \tau(x)$, where $\tau(x)=\frac{f(x)}{F(x)}$ and

$$
g(x)=\frac{\left(x^{2}+1\right)^{2 n} P(x)}{2 x^{2 n-3}\left(x^{2}-1\right)^{2}},
$$

where $P(x)$ is given by Eq. (4.3), if and only if

$$
f(x)=2 \frac{\Gamma(2 n)}{\Gamma(n) \Gamma(n-1)} \frac{x^{2 n-3}\left(x^{2}-1\right)^{2}}{\left(x^{2}+1\right)^{2 n}}, x>1, n \geq 2 .
$$

Proof. It is known from see Ahsanullah, et al. (2013) and David and Nagaraja (2003) that $E\left(S_{k-1} \mid X_{k, n}=x\right)=$ $E(X \mid X \leq x)$. Hence, by Theorem 4.1, the result follows.

Theorem 4.4: Suppose the random variable $X$ satisfies the Assumption 4.1 with $\gamma=1$ and $\delta=\infty$, then $E\left(T_{k, n} \mid X_{k, n}=x\right)=h(x) r(x)$, where $r(x)=\frac{f(x)}{1-F(x)}$ and

$$
h(x)=\frac{\Gamma(n) \Gamma(n-1)\left(x^{2}+1\right)^{2 n}\left[E(x)-\frac{\Gamma(2 n)}{\Gamma(n) \Gamma(n-1)} P(x)\right]}{2 \Gamma(2 n) x^{2 n-3}\left(x^{2}-1\right)},
$$

if and only if

$$
f(x)=2 \frac{\Gamma(2 n)}{\Gamma(n) \Gamma(n-1)} \frac{x^{2 n-3}\left(x^{2}-1\right)^{2}}{\left(x^{2}+1\right)^{2 n}}, x>1, n \geq 2,
$$

where $E(x)$ denotes the first moment given by Eq. (3.6), and $P(x)$ is given by Eq. (4.3).

Proof. It is known from see Ahsanullah, et al. (2013) and David and Nagaraja (2009) that $E\left(T_{k, n} \mid X_{k, n}=x\right)=$ $E(X \mid X \geq x)$. Hence the proof follows from Theorem 4.2.

\subsection{Characterization by Upper Record Values}

For details on record values, see Ahsanullah (1995). Let $X_{1}, X_{2}, \ldots$ be a sequence of independent and identically distributed absolutely continuous random variables with distribution function $F(x)$ and pdf $f(x)$. If $Y_{n}=\max \left(X_{1}, X_{2}, \ldots, X_{n}\right)$ for $n \geq 1$ and $Y_{j}>Y_{j-1}, j>1$, then $X_{j}$ is called an upper record value of $\left\{X_{n}, n \geq 1\right\}$. The indices at which the upper records occur are given by the record times $\left\{U(n)>\min \left(j \mid j>U(n+1)\right.\right.$., $X_{j}>$ $\left.\left.X_{U(n-1)}, . n>1\right)\right\}$ and $U(1)=1$. Let the $n$th upper record value be denoted by $X(n)=X_{U(n)}$.

Theorem 4.5: Suppose the random variable $X$ satisfies the Assumption 4.1 with $\gamma=1$ and $\delta=\infty$, then $E(X(n+1) \mid X(n)=x)=h(x) r(x)$, where $r(x)=\frac{f(x)}{1-F(x)}$ and

$$
h(x)=\frac{\Gamma(n) \Gamma(n-1)\left(x^{2}+1\right)^{2 n}\left[E(x)-\frac{\Gamma(2 n)}{\Gamma(n) \Gamma(n-1)} P(x)\right]}{2 \Gamma(2 n) x^{2 n-3}\left(x^{2}-1\right)},
$$


if and only if

$$
f(x)=2 \frac{\Gamma(2 n)}{\Gamma(n) \Gamma(n-1)} \frac{x^{2 n-3}\left(x^{2}-1\right)^{2}}{\left(x^{2}+1\right)^{2 n}}, x>1, n \geq 2,
$$

where $E(x)$ denotes the first moment given by Eq. (3.6), and $P(x)$ is given by Eq. (4.3).

Proof. It is known from Ahsanullah, et al. (2013) and Nevzorov (2001) that $E(X(n+1) \mid X(n)=x)=E(X \mid X \geq x)$. Then, the result is evident from Theorem 4.2.

\section{Percentiles}

For any statistical applications, it is important to know the percentage points of a given distribution. For example, one may be interested in knowing the median (50\%), $25 \%$, or $75 \%$ quartiles, or $5 \%, 90 \%$, $95 \%$, or 99\% confidence levels for other applications, to assess the statistical significance of an observation whose distribution is known.

The 100pth percentile or the quantile of order $p$, for any $0<p<1$, of our distribution with the $\operatorname{pdf} f_{X}(x)$ as in Eq. (3.1) is defined as a number $x_{p}$ such that the area under $f_{X}(x)$ to the left of $x_{p}$ is $p$. In other words, $x_{p}$ is any solution of the equation $F\left(x_{p}\right)=\int_{1}^{x_{p}} f_{X}(u) d u=p$, where $F\left(x_{p}\right)$ denotes the cdf given by Eq. (3.6). Thus, by numerically solving the equation $F\left(x_{p}\right)=\int_{1}^{x_{p}} f_{X}(u) d u=p$, using a Maple program, percentage points $x_{p}$ associated with the cdf, $F\left(x_{p}\right)$, of $X$ are computed for different sets of values of the parameters, $n \geq 2$, which are provided in the Table 3 below.

Table 3: Percentage Points

\begin{tabular}{ccccccc}
\hline Percentiles $n$ & $\mathbf{0 . 7 5}(\mathbf{7 5} \%)$ & $\mathbf{0 . 8 0}(\mathbf{8 0} \%)$ & $\mathbf{0 . 8 5}(\mathbf{8 5} \%)$ & $\mathbf{0 . 9 0}(\mathbf{9 0} \%)$ & $\mathbf{0 . 9 5}(\mathbf{9 5} \%)$ & $\mathbf{0 . 9 9}(\mathbf{9 9} \%)$ \\
\hline $\mathbf{2}$ & 4.5686 & 5.1866 & 6.0768 & 7.5467 & 10.8156 & 24.4335 \\
$\mathbf{3}$ & 2.8311 & 3.0573 & 3.3589 & 3.8084 & 4.6613 & 7.2155 \\
$\mathbf{4}$ & 2.3201 & 2.4617 & 2.6452 & 2.9085 & 3.3803 & 4.6382 \\
$\mathbf{5}$ & 2.0659 & 2.1714 & 2.3058 & 2.4950 & 2.8238 & 3.6485 \\
$\mathbf{6}$ & 1.9103 & 1.9956 & 2.1034 & 2.2530 & 2.5082 & 3.1242 \\
$\mathbf{7}$ & 1.8040 & 1.8762 & 1.9671 & 2.0921 & 2.3025 & 2.7973 \\
$\mathbf{8}$ & 1.7254 & 1.7889 & 1.8680 & 1.9763 & 2.1567 & 2.5725 \\
$\mathbf{9}$ & 1.6649 & 1.7217 & 1.7923 & 1.8884 & 2.0471 & 2.4076 \\
$\mathbf{1 0}$ & 1.6165 & 1.6682 & 1.7322 & 1.8189 & 1.9613 & 2.2809 \\
$\mathbf{1 1}$ & 1.5768 & 1.6243 & 1.6831 & 1.7624 & 1.8920 & 2.1800 \\
$\mathbf{1 2}$ & 1.5434 & 1.5876 & 1.6421 & 1.7154 & 1.8347 & 2.0976 \\
\hline
\end{tabular}

\section{Concluding Remarks}

The condition numbers of Gaussian matrices occur in many branches of pure and applied sciences, and engineering. Many researchers have studied the distributions of condition numbers of Gaussian matrices. In this paper, several new distributional properties of the distribution of the condition number of a complex Gaussian matrix are presented. Based on the distributional properties, we have established some new characterization results. For some values of the parameter, $n \geq 2$, the first moment, $E(X)$, and the Shannon entropy are numerically computed. Since, for any statistical applications, it is important to know the percentage points of a given distribution, we also have computed the percentiles of the distribution. It is hoped that the findings of this paper will be quite useful for researchers in various fields of pure and applied 
sciences.

Acknowledgements: The authors are thankful to the editor-in-chief and the referees for their valuable comments and suggestions, which certainly improved the presentation of the paper. Finally, the authors are also thankful to Professor Dr. R.C. Singh Chandel, (Professor of Mathematics, D.V. Postgraduate College, Orai, U.P., India, and Executive Editor, Jñânâbha, Vijñâna Parishad of India) for his valuable advice to bring the paper in its present form.

\section{References}

[1] Ahsanullah, M. (1995). Record Statistics. Nova Science Publishers, New York, USA.

[2] Abramowitz, M., and Stegun, I. A. (1970). Handbook of Mathematical Functions, with Formulas, Graphs, and Mathematical Tables. Dover, New York, USA.

[3] Acosta, G., Grana, M., and Pinasco, J. P. (2006). Condition numbers and scale free graphs. The European Physical Journal B-Condensed Matter and Complex Systems, 53 (3), 381 - 385.

[4] Anderson,W., and Wells, M. T. (2009). The exact distribution of the condition number of a Gaussianmatrix. SIAM Journal on Matrix Analysis and Applications, 31 (3), 1125 - 1130.

[5] Ahsanullah, M., and Shakil, M. (2018). Some characterizations of raised cosine distribution. International Journal of Advanced Statistics and Probability, 6(2), 42 - 49.

[6] Ahsanullah, M., Shakil, M., and Kibria, B. G. (2015). Characterizations of folded student's t distribution. Journal of Statistical Distributions and Applications, 2(1), 15.

[7] Ahsanullah, M., Shakil, M., and Kibria, B. M. (2016). Characterizations of Continuous Distributions by Truncated Moment. Journal of Modern Applied Statistical Methods, 15(1), 17 - 20.

[8] Ahsanullah, M., Nevzorov, V. B., and Shakil, M. (2013). An Introduction to Order Statistics. Atlantis-Press, Paris, France.

[9] Arnold, B.C., Balakrishnan, N., and Nagaraja, H. N. (2005). First Course in Order Statistics. Wiley, New York, USA.

[10] Bottcher, A., and Grudsky, S. M. (1998). On the Condition Numbers of Large Semi-definite Toeplitz Matrices. Linear Algebra and its Applications, 279, $285-301$.

[11] Chen, Z., and Dongarra, J. J. (2005). Condition numbers of Gaussian random matrices. SIAM Journal on Matrix Analysis and Applications, 27(3), $603-620$.

[12] Datta, B. N. (1995), Numerical Linear Algebra and Applications, Brooks/Cole Publishing Company, California, USA.

[13] David, H. A., and Nagaraja, H. N. (2003). Order Statistics. Third Edition, Wiley, New York, USA.

[14] Demmel, J. W. (1987). On condition numbers and the distance to the nearest ill-posed problem. Numerische Mathematik, 51 (3), 251 - 289.

[15] Demmel, J. W. (1988). The probability that a numerical analysis problem is difficult. Mathematics of Computation, 50 (182), $449-480$

[16] Deng, X., López-Martínez, C., Jinsong Chen, J., and Han, P. (2017). Statistical Modeling of Polarimetric SAR Data: A Survey and Challenges. Remote Sensing, 9(4), 348

[17] Edelman, A. (1988). Eigenvalues and condition numbers of random matrices. SIAM Journal on Matrix Analysis and Applications, 9 (4), $543-560$.

[18] Edelman, A. (1992). On the distribution of a scaled condition number. Mathematics of Computation, 58(197), 185 - 190. 35

[19] Edelman, A., and Rao, N. R. (2005). Random matrix theory. Acta Numerica, 14, 233 - 297.

[20] Edelman, A., and Wang, Y. (2013). Random matrix theory and its innovative applications. In Advances in Applied Mathematics, Modeling, and Computational Science, 91 - 116. Springer, USA.

[21] Ern, A., and Guermond, J. L. (2006). Evaluation of the condition number in linear systems arising in finite element approximations. ESAIM: Mathematical Modelling and Numerical Analysis, 40 (1), 29 - 48.

[22] Galambos, J., and Kotz, S. (1978). Characterizations of probability distributions. A unified approach with an emphasis on exponential and related models. Lecture Notes in Mathematics, 675, Springer, Berlin, Germany.

[23] Gradshteyn, I. S., and Ryzhik, I. M. (1990). Table of integrals, series, and products, Academic Press, Inc., San Diego, California, USA.

[24] Geurts, A. J. (1982). A contribution to the theory of condition. Numerische Mathematik, 39 (1), 85 - 96.

[25] Golub, G. H., and Van Loan, C. F. (1996), Matrix Computations, Third Edition, Johns Hopkins University Press, Baltimore.

[26] Haagerup, U., and Thorbjørnsen, S. (2003). Random matrices with complex Gaussian entries. Expositiones Mathematicae, 21(4), 293 - 337.

[27] Hargreaves, G. I. (2005). Computing the condition number of tridiagonal and diagonal-plus-semiseparable matrices in linear time. SIAM journal on matrix analysis and applications, 27 (3), 801 - 820. 
[28] Higham, N. J. (2002). Accuracy and Stability of Numerical Algorithms, Second Edition, Society for Industrial and Applied Mathematics, Philadelphia, PA, USA.

[29] Kotz, S., and Shanbhag, D. N. (1980). Some new approaches to probability distributions. Advances in Applied Probability, $12,903-921$.

[30] Lei, Y. J., Xu, W. R., Lu, Y., Niu, Y. R., and Gu, X. M. (2014). On the symmetric doubly stochastic inverse eigenvalue problem. Linear Algebra and its Applications, 445, 181 - 205.

[31] Leon, S. J. (2006), Linear Algebra with Applications, Prentice Hall, New Jersey, USA.

[32] Nagaraja, H. N. (2006). Characterizations of Probability Distributions, In Springer Handbook of Engineering Statistics (pp. 79 - 95), Springer, London, UK.

[33] Nevzorov, V. B. (2001). Records: Mathematical Theory, Translation of Mathematical Monograph. American Mathematical Society, Rhode Island, USA.

[34] Rice, J. R. (1966). A theory of condition. SIAM Journal on Numerical Analysis, 3 (2), 287 - 310.

[35] Shakil, M., and Ahsanullah, M. (2016). Characterizations of the distribution of the Demmel condition number of real Wishart matrices. Special Matrices, 4(1), 352 - 365.

[36] Shakil, M., and Ahsanullah, M. (2017). Some inferences on the distribution of the Demmel condition number of complex Wishart matrices. Special Matrices, 5(1), 127 - 138.

[37] Shakil, M., and Ahsanullah, M. (2018). A note on the characterizations of the distributions of the condition numbers of real Gaussian matrices. Special Matrices, 6(1), 282 - 296.

[38] Shi, L., Gan, T., Zhu, H., and Gu, X. (2013). The exact distribution of the condition number of complex random matrices, The Scientific World Journal, Article ID 729839, 4 pages, DOI: 10.1155/2013/729839.

[39] Skeel, R. D. (1979). Scaling for numerical stability in Gaussian elimination. Journal of the The Association for Computing Machinery (JACM), 26 (3), 494 - 526.

[40] Shannon, C. E. (1948). A mathematical theory of communication. Bell system technical journal, 27 (3), 379 - 423.

[41] Trefethen, L. N., and Bau, D. (1997). Numerical Linear Algebra, Society for Industrial and Applied Mathematics, Philadelphia, PA, USA.

[42] Viswanath, D., and Trefethen, L. N. (1998). Condition numbers of random triangular matrices. SIAM Journal on Matrix Analysis and Applications, 19 (2), 564 - 581.

[43] Turing, A. M. (1948). Rounding-off errors in matrix processes. The Quarterly Journal of Mechanics and Applied Mathematics, 1 (1), $287-308$.

[44] Wei, L., McKay, M. R., and Tirkkonen, O. (2011). Exact Demmel condition number distribution of complex Wishart matrices via the Mellin transform. IEEE Communications Letters, 15(2), 175 - 177.

[45] Wigner, E. (1955). Characteristic Vectors of Bordered Matrices with Infinite Dimensions. Annals of Mathematics, 62 (3), 548 $-564$.

[46] Wishart, J. (1928). The generalised product moment distribution in samples from a normal multivariate population. Biometrika, 32 - 52.

[47] Xu, S., and Zhang, J. (2004). A New Data Mining Approach to Predicting Matrix Condition NumbersCommunications in Information and Systems, 4 (4), $325-340$.

[48] Zhang, W., Wang, C. X., Tao, X., and Patcharamaneepakorn, P. (2016). Exact distributions of finite random matrices and their applications to spectrum sensing. Sensors, 16 (8), 1183.

[49] Zhong, C., McKay, M. R., Ratnarajah, T., and Wong, K. K. (2011). Distribution of the Demmel condition number of Wishart matrices. IEEE Transactions on Communications, 59 (5), 1309 - 1320. 